\title{
Si(775)-Au atomic chains: Geometry, optical properties, and spin order
}

\author{
Christian Braun, ${ }^{1, *}$ Conor Hogan,${ }^{2,3}$ Sandhya Chandola, ${ }^{4}$ Norbert Esser, ${ }^{4}$ Simone Sanna, ${ }^{5}$ and Wolf Gero Schmidt ${ }^{1}$ \\ ${ }^{1}$ Lehrstuhl für Theoretische Materialphysik, Universität Paderborn, 33095 Paderborn, Germany \\ ${ }^{2}$ Istituto di Struttura della Materia-CNR (ISM-CNR), Via Fosso del Cavaliere 100, 00133 Rome, Italy \\ ${ }^{3}$ Dipartimento di Fisica, Universitá di Roma “Tor Vergata”, Via della Ricerca Scientifica 1, 00133 Rome, Italy \\ ${ }^{4}$ Leibniz-Institut für Analytische Wissenschaften - ISAS - e.V., Schwarzschildstraße 8, 12489 Berlin, Germany \\ ${ }^{5}$ Institut für Theoretische Physik, Universität Giessen, Heinrich-Buff-Ring 16, 35392 Gießen, Germany
}

(Received 19 July 2017; published 23 October 2017)

\begin{abstract}
The geometry and electronic structure of self-assembled atomic scale $\mathrm{Au}$ wires on $\mathrm{Si}(775)$ are investigated within density functional theory. The calculated surface diagram indicates the existence of two stable configurations with Au coverages of 0.32 and 0.96 monolayers, respectively. The low-coverage structure is predicted to host an antiferromagnetic spin chain localized at the $\mathrm{Si}$ rest atom dangling bonds, while the high-coverage structure is characterized by a Au-induced $\beta-\sqrt{3}$-like structure on the terrace. These structural models are supported by the comparison of measured and calculated surface optical anisotropies.
\end{abstract}

DOI: 10.1103/PhysRevMaterials.1.055002

\section{INTRODUCTION}

Self-assembled metallic nanowires on semiconductor surfaces are popular model systems to explore peculiarities of low-dimensional physics such as Peierls instabilities [1-4], Luttinger liquids [5], or solitons [6]. Gold-induced wire structures on $\mathrm{Si}(553)$ and $\mathrm{Si}(557)$ are being discussed as possible hosts for spin chains $[7,8]$. Spin-ordered structures in nonmagnetic materials allow for local magnetic moments without a perturbing magnetic surrounding. The identification of further spin-ordered wire structures as well as the understanding of the preconditions for the formation of spin order in low-dimensional structures are therefore of recent interest [9].

Here we use density functional theory (DFT) to explore the geometry and possible spin polarization effects in $\mathrm{Si}(775)$ $\mathrm{Au}$ surfaces. The Au-induced atomic scale wires on $\mathrm{Si}(775)$ belong to the large group of quasi-one-dimensional structures obtained by Au deposition onto stepped silicon surfaces [10]. Compared to other Au-induced chain structures on Si surfaces (see, e.g., Refs. [7,8,11,12]), relatively little information is available on the geometry and electronic structure of $\mathrm{Si}(775)$ $\mathrm{Au}[9,10]$. Here, total-energy calculations for a large number of plausible structural configurations for various Au coverages are performed. Two stable geometries are predicted. Surface optical spectroscopy experiments and simulations are shown to support the predicted structural models. Finally, the electronic structure and spin order of the most stable geometries are explored in detail. Unlike other $\mathrm{Si}(h h k)$-Au surfaces, which exhibit spin ordering at the step edge, $\mathrm{Si}(775)$-Au is shown to host spin chains lying on the terraces.

\section{METHODOLOGY}

The Vienna $a b$ initio simulation package (VASP) [13] implementation of DFT is used to determine the structural

\footnotetext{
*braun91@mail.uni-paderborn.de

Published by the American Physical Society under the terms of the Creative Commons Attribution 4.0 International license. Further distribution of this work must maintain attribution to the author(s) and the published article's title, journal citation, and DOI.
}

and electronic properties of $\mathrm{Si}(775)-\mathrm{Au}$. The electron-electron exchange and correlation effects are modeled within the generalized gradient approximation (GGA) using the PBE functional [14]. Calculations within the local-density approximation (LDA) are performed for comparison. Projected augmented waves [15] are used for the description of the ion-electron interaction. The $\mathrm{Si}(775)$-Au surfaces are modeled within periodic supercells, consisting of six essentially bulklike Si bilayers stacked along the surface normal. An additional layer contains the $\mathrm{Au}$ and $\mathrm{Si}$ surface atoms. The bottom layer $\mathrm{Si}$ atoms are passivated by hydrogen atoms. A vacuum region of $25 \AA$ decouples the slab from its periodic images along the surface normal. The lowest two Si bilayers are kept fixed in ideal bulk positions during structural relaxation, while all remaining atoms are free to move. The electron orbitals are expanded into plane waves up to an energy cutoff of $410 \mathrm{eV}$. The Brillouin zone is sampled using a $2 \times 7 \times 1 k$-point mesh. Spin-polarized calculations are made assuming collinear spin orientation. Simulated STM images are calculated using the Tersoff-Hamann model [16].

Optical spectra were computed for selected models using the Yambo code [17]. The surface and bulk response functions were computed in the independent particle (IP) approximation based on the DFT electronic structure generated with Quantum ESPRESSO [18] using the geometries obtained from the VASP calculations. Norm-conserving pseudopotentials and a plane wave cutoff of $820 \mathrm{eV}$ were used. Regular, dense $k$-point meshes containing 192 and $128 k$ points in the full Brillouin zone were used for $(1 \times 2)$ and $(1 \times 3)$ cells, respectively. Reflectance anisotropy spectroscopy (RAS) signals were then computed (see, e.g., Refs. [19,20] for more details) from the polarizabilities $\alpha_{i i}$ of the $\mathrm{Si} / \mathrm{Au}$ slabs and the dielectric function of bulk silicon $\epsilon_{b}$ :

$$
\frac{\Delta R}{R}=\frac{16 \pi \omega d}{c} \operatorname{Im} \frac{\alpha_{[1 \overline{1} 0]}(\omega)-\alpha_{[11 \overline{2}]}(\omega)}{\epsilon_{b}(\omega)-1}
$$

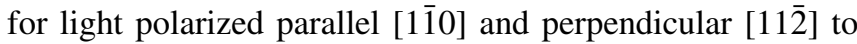
the step edges; $d$ is the slab thickness. The use of LDA/PBE eigenvalues in an IP approach generally results in spectra that are redshifted with respect to experiment. 
Finally, RAS measurements were performed on the $\mathrm{Si}(775)$ surface for different Au coverages. The $n$-type $\operatorname{Si}(775)$ substrate was cleaned by direct current heating up to $1200^{\circ} \mathrm{C}$. To prepare the standard (low Au coverage of $0.32 \mathrm{ML}$ ) reconstruction of $\mathrm{Si}(775)-\mathrm{Au}$, the sample was held at $650^{\circ} \mathrm{C}$ during $\mathrm{Au}$ evaporation. Low energy electron diffraction (LEED) and RAS transients were used to check the sample preparation. All measurements were performed at room temperature.

\section{RESULTS}

\section{A. Geometries}

The Si(775)-Au surface differs from vicinal surfaces with smaller indices such as $\mathrm{Si}(553)-\mathrm{Au}$ or $\mathrm{Si}(557)-\mathrm{Au}$ by its shallower cutting angle of $8.5^{\circ}$. This leads to comparatively broad terraces of $21.3 \AA$ [10]. Compared to the Si(553)-Au surface, this is an increase of about $44 \%$ in terrace width, thus providing considerably more space for various surface reconstruction motifs, such as adatom/rest-atom rows, multiple $\mathrm{Au}$ chains, and Si honeycomb chains. Honeycomb chains (HCs) have been found to characterize most, if not all, Au-induced reconstructions on stepped Si surfaces [8-10,21]. They can therefore be expected to form also on $\mathrm{Si}(775)-\mathrm{Au}$ for gold coverages far below 1 monolayer (ML) and are considered here in many structural variants. Due to the larger terraces, broad "honeycomb ribbons" are considered, as well as narrow zigzag structures (i.e., Seiwatz chains [22]). Surface atom substitution and adsorption rather than diffusion into the surface are observed for Au-deposited $\mathrm{Si}$ surfaces [23] and are explored here computationally. The atomic Au chains typically bridge the $\mathrm{HC}$ at the step edge. Their position and width are therefore crucial for determining the correct surface reconstruction. Here $\mathrm{Si}(775)$-Au surface models containing between four and seven $\mathrm{Au}$ atoms per $(1 \times 2)$ surface cell, corresponding to Au coverages of 0.32 , $0.40,0.48$, and $0.56 \mathrm{ML}$, are probed with respect to (i) the position of the $\mathrm{Au}$ atoms on the terraces, (ii) their geometry, e.g., dimerized double strand, zigzag chain (cf. Figs. 1 and 3 in Ref. [21]), (iii) the width of the HC, and (iv) the number and (v) the position of possible Si adatoms. In addition, high-coverage structures drawn from $\mathrm{Si}(111)$-Au studies [e.g., $\left.\operatorname{Si}(111)-(\sqrt{3} \times \sqrt{3}) R 30^{\circ}-\mathrm{Au}\right]$ are explored, yielding $0.96 \mathrm{ML}$ $\mathrm{Au}$ reconstructions having $18 \mathrm{Au}$ atoms per $(1 \times 3)$ surface cell. Altogether, more than one hundred different models have been explored [24]. The models were structurally relaxed and compared energetically assuming a $\mathrm{Si}$ chemical potential equal to its bulk value, $\mu_{\mathrm{Si}}^{\text {bulk }}$. The most stable structures corresponding to the respective Au coverage are shown in Fig. 1.

In the case of a $0.32 \mathrm{ML}$ Au coverage, a model comprising a dimerized double strand Au chain next to a Si HC at the step edge, with a single $\mathrm{Si}$ adatom and rest atom per $(1 \times 2)$ surface unit cell, is stable, as depicted in Fig. 1(a). It corresponds to the structure investigated by Aulbach et al. [9]. All variants with modified Au chains, step edge geometries, and adatom sites lead to a less stable configuration. All stable $\mathrm{Si}(775)-\mathrm{Au}$ surfaces with gold coverages up to $0.56 \mathrm{ML}$ feature atomic gold wires in the form of dimerized double strand chains, typical for $\operatorname{Si}(h h k)$ surfaces with $h>k$, next to a HC. The stability
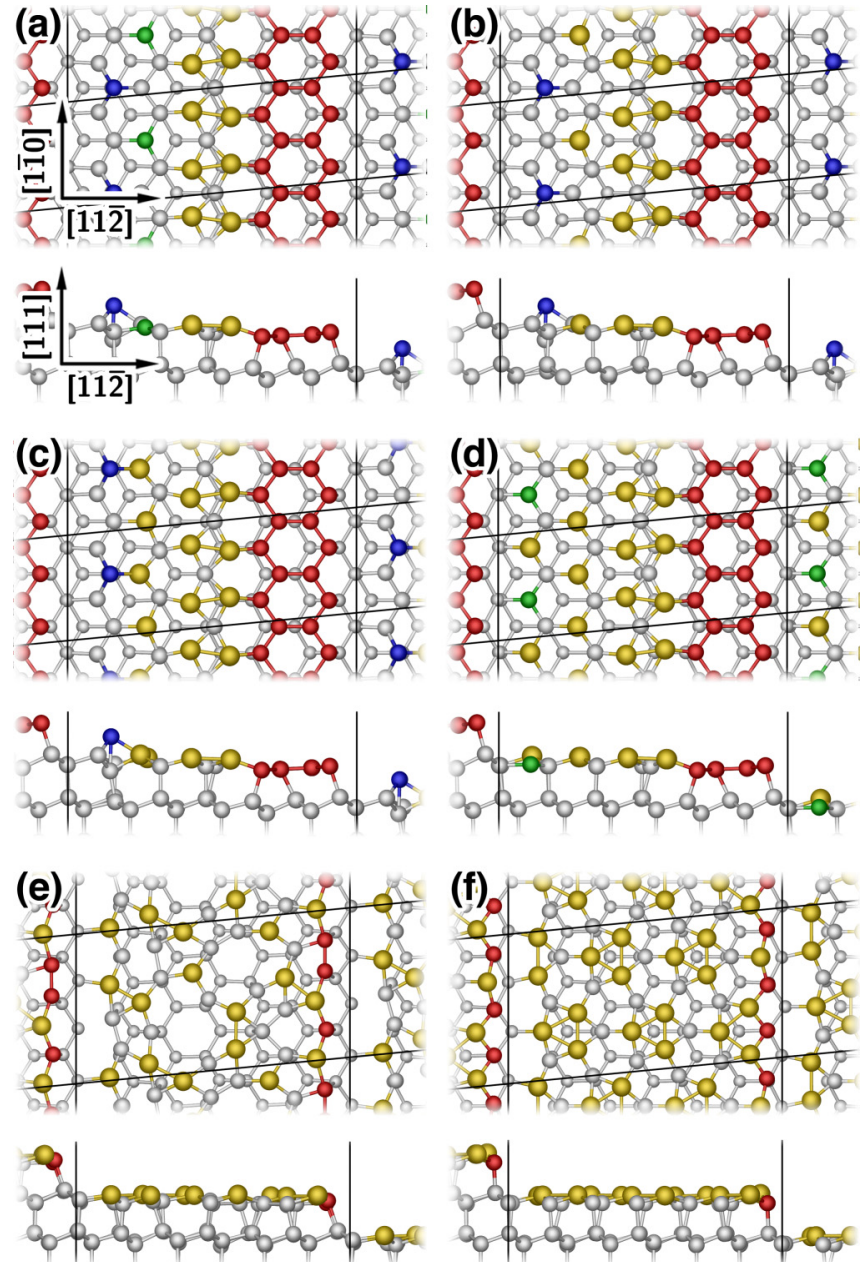

FIG. 1. Top and side view of the energetically most favored $\mathrm{Si}(775)-\mathrm{Au}$ surface structures for $\mathrm{Au}$ coverages of (a) $0.32 \mathrm{ML}$ ("dbl-Au"), (b) $0.40 \mathrm{ML}$, (c) $0.48 \mathrm{ML}$, (d) $0.56 \mathrm{ML}$, (e) $0.64 \mathrm{ML}$ $[\alpha-(\sqrt{3} \times \sqrt{3})]$, and (f) $0.96 \mathrm{ML}[\beta-(\sqrt{3} \times \sqrt{3})]$. The color coding is $\mathrm{Au}$ atoms (yellow), Si HC and step edge atoms (red), Si adatoms (blue), and $\mathrm{Si}$ rest atoms (green).

of a dimerized double strand in comparison to zigzag gold chains can be understood as a dislocation formation along the $[11 \overline{2}]$ direction (see Fig. 1). This dislocation occurs for $\mathrm{Si}(h h k)$ surfaces with $h>k$ due to the formation of the HC and is normally situated between the $\mathrm{HC}$ and neighboring $\mathrm{Si}$ atoms or Au atoms, respectively. The dimerized double strand chain, in contrast to the zigzag chain, is incorporated in this dislocation in such a way that the $\mathrm{Si}$ atoms adjacent to the $\mathrm{Au}$ chain experience a bulklike coordination.

Additional $\mathrm{Au}$ atoms deposited on the surface tend to substitute rest atoms in the vicinity of the Au chain. Substitution is dominant: no adsorption without substitution near or on top of the original chain was observed. This leads to wide atomic wires or even broad $\mathrm{Si}$-Au "stripes" spanning almost the whole terrace as in the case of $0.56 \mathrm{ML}$ Au coverage. This is in contrast to high Au coverage structures on $\mathrm{Si}(111)-(5 \times 2)-\mathrm{Au}$ where an additional $\mathrm{Au}$ atom is adsorbed on top of existing triple Au chains [12]. Remaining Si rest atoms that are not substituted by $\mathrm{Au}$ are saturated by $\mathrm{Si}$ adatoms whenever the number of rest atoms is reduced by at least two by the adatom. 


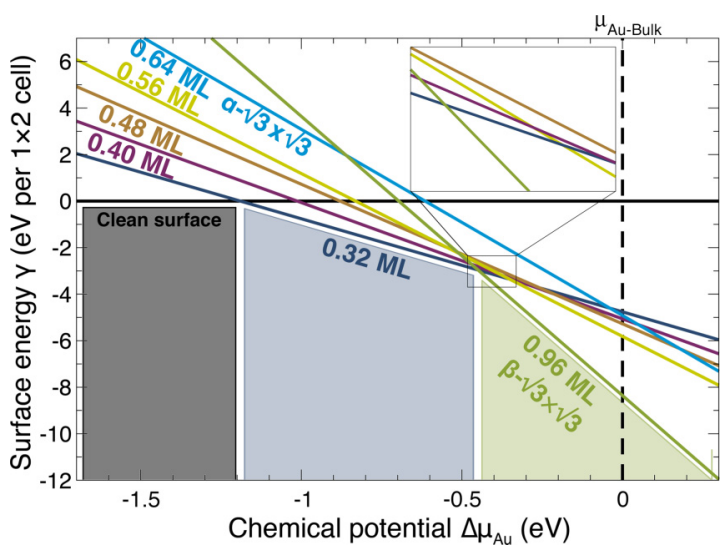

FIG. 2. Calculated surface phase diagram of $\mathrm{Si}(775)-\mathrm{Au}$ as a function of the Au chemical potential $\Delta \mu_{\mathrm{Au}}$ relative to its bulk value. Regions of stability for low and high coverage phases are indicated.

In the case of 0.40 ML Au coverage [see Fig. 1(b)], no rest atoms are present on the surface as the three remaining rest atoms per $(1 \times 2)$ cell are passivated by a single $\mathrm{Si}$ adatom. The same holds for the $0.48 \mathrm{ML}$ covered surface in Fig. 1(c), except that the $\mathrm{Si}$ adatom forms bonds with two remaining rest atoms and a single $\mathrm{Au}$ atom. At this coverage, the gold atoms are arranged in a triple chain structure, composed of a single and double dimerized row, adjacent to the Si honeycomb. This geometry is thus similar to that proposed for low Au coverages on $\mathrm{Si}(111)-(5 \times 2)-\mathrm{Au}[11,23]$. The $0.56 \mathrm{ML} \mathrm{Au}$ decorated surface in Fig. 1(d) instead exhibits no Si adatoms, as saturation of a single rest atom by an adatom is not favorable.

For high-coverage structures, $\operatorname{Si}(111)-(\sqrt{3} \times \sqrt{3}) R 30^{\circ}-\mathrm{Au}$ structures superimposed on $\mathrm{Si}(775)$ terraces were probed. We focused on the honeycomb configuration $\left(\mathrm{H}_{3}\right.$-MTL) [25] and the conjugate honeycomb-chained trimer (CHCT) [26] model for $\alpha-(\sqrt{3} \times \sqrt{3})$ and $\beta-(\sqrt{3} \times \sqrt{3})$, respectively [see Figs. 1(e) and 1(f)]. Depending on the step edge reconstruction, the $\alpha-\sqrt{3}$ comprises a Au coverage of up to $0.64 \mathrm{ML}$ and the $\beta-\sqrt{3}$ of up to 0.96 ML. Due to the nature of the $(\sqrt{3} \times \sqrt{3})$ substructure, the smallest surface unit cell has a $(1 \times 3)$ periodicity.

The described models constitute the most stable ones identified for specific Au coverages. The grand canonical potential (see, e.g., Refs. [27,28]) has been calculated in order to compare the overall thermodynamic stability as a function of the Au chemical potential. The resulting phase diagram is shown in Fig. 2. Formation energies are plotted relative to that of the ideal, clean $\mathrm{Si}(775)$ surface. The clean $\mathrm{Si}$ surface is stable for low values of the gold chemical potential. More Au-rich preparation conditions increase the Au chemical potential and stabilize the $\mathrm{Au}$ atomic wires with $0.32 \mathrm{ML}$. Interestingly, further increase of the Au chemical potential does not lead to broader atomic wires, but directly to the $\beta-\sqrt{3}$ structure. The $0.40-0.56 \mathrm{ML}$ structures identified above are metastable and do not correspond to the thermodynamic ground state. The same holds for the $\alpha-\sqrt{3}$ structure, which is significantly less stable than the remaining structures. These findings do not change qualitatively when the calculations are performed within the DFT-LDA. We can thus conclude that there are only two relevant $\mathrm{Si}(775)-\mathrm{Au}$ geometries, i.e., the
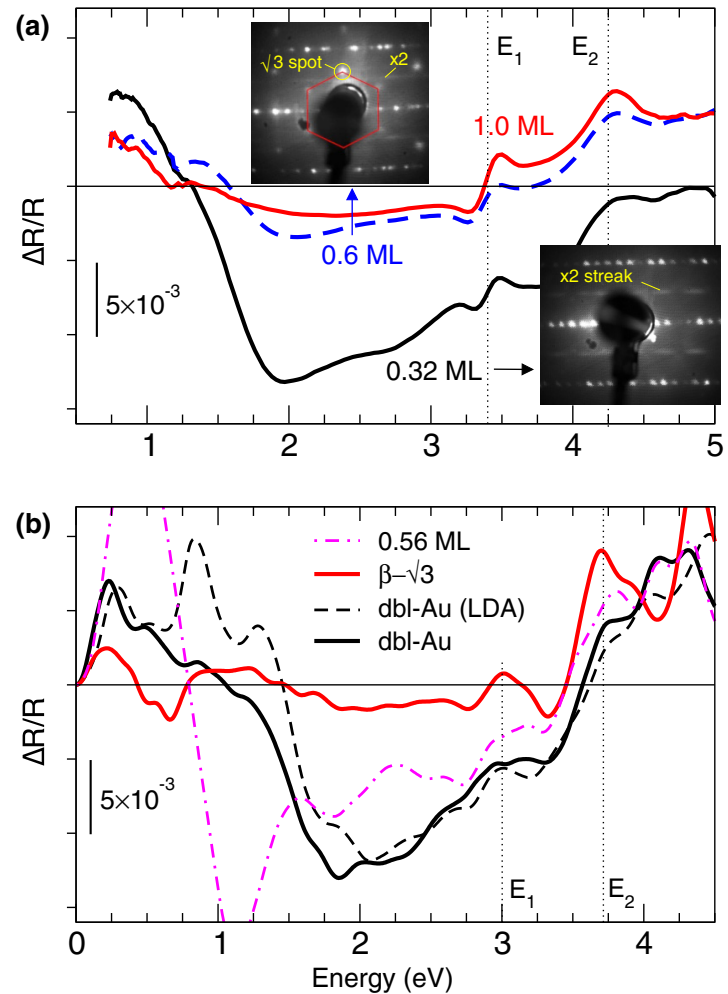

FIG. 3. Reflectance anisotropy spectra of the $\mathrm{Si}(775)$-Au surface. (a) Experimental data measured at room temperature for $0.32,0.6$, and 1.0 ML coverages of $\mathrm{Au}$, along with (inset) LEED images for the 0.32 and $0.6 \mathrm{ML}$ coverages. (b) Calculated spectra for the dbl-Au and $\beta-\sqrt{3}$ reconstructions, as well as for the most stable $0.56 \mathrm{ML}$ model [see Fig. 1(d)]. All spectra are computed within PBE; the LDA spectrum for dbl-Au is also shown.

atomic wire structure shown in Fig. 1(a) (referred to henceforth as "dbl-Au") and the $\beta-\sqrt{3}$ phase shown in Fig. 1(f), with coverages of 0.32 and $0.96 \mathrm{ML}$, respectively.

\section{B. Optical properties}

Comparison of measured and calculated reflectance anisotropy spectra has been very helpful for identifying or discarding surface structural models [20]. RAS is particularly adept at distinguishing between similar quasi-1D surface reconstructions [29] and has previously been applied with success in the study of nominal $\mathrm{Si}(111)$-Au and other vicinal $\mathrm{Si}(h h k)$-Au systems [26,30-32]. We thus carried out new experimental measurements of the RAS spectra of $\mathrm{Si}(775)-\mathrm{Au}$ for different gold coverages and compared with calculations for the most stable models predicted in the previous section.

Experimental results are shown in Fig. 3(a). The data for the 0.32 ML surface compares favorably with previously published measurements on this surface [33]. The spectrum exhibits a fairly weak positive peak at $1 \mathrm{eV}$ and a broader negative feature in the 1.5 to $4.2 \mathrm{eV}$ range. Qualitatively similar spectra have been observed on $\mathrm{Si}(557)-\mathrm{Au}, \mathrm{Si}(553)-\mathrm{Au}$, and $\mathrm{Si}(111)-\mathrm{Au}[32,33]$. For this class of surfaces, it is known that the $1.5-3.0 \mathrm{eV}$ negative structure derives from transitions involving Si honeycomb chains. The positive low energy feature instead has a more complicated character, being linked 
to various transitions associated with states delocalized along the Au chains and at the step edge dangling bonds [30,31]. The corresponding LEED pattern (see figure inset) reveals the presence of $\times 2$ streaks, arising from the dimerization of the Au chains. The spectrum shows an oscillation around the bulk $E_{1}$ energy and a peak at $E_{2}$, reflecting the perturbation of the anisotropic surface potential on the bulk states of the $\mathrm{Si}$ substrate.

At a slightly higher coverage of $0.45 \mathrm{ML}$, the LEED signal (not shown) already indicates the substantial growth of a second phase via the presence of additional (weak) spots: this surface is composed of a 50:50 mixture of a $\sqrt{3}$ phase in addition to the $\times 2$ phase. By 0.6 ML, the LEED (see inset) shows intense $\sqrt{3}$ spots $(\sim 60 \%$ of surface $)$ as well as the continued presence of the $\times 2$ phase $(40 \%)$. With respect to the pure $\times 2$ phase, the RAS line shape above $1.5 \mathrm{eV}$ is broad and attenuated, indicating that the additional $\mathrm{Au}$ has disturbed the honeycomb structure and quenched transitions related to it. There is now a small broad positive peak at $1.3 \mathrm{eV}$, and a weak increasing feature at the lower energy limit of measurement, possibly indicating an anisotropic Drude-like term. At a much higher coverage of about $1 \mathrm{ML}$, the RAS response between $1.1 \mathrm{eV}$ and $3.4 \mathrm{eV}$ is rather flat, indicating that the HC-related transitions are almost all quenched. The combination of LEED and RAS measurements therefore indicates the existence of two (and only two) distinct phases on $\mathrm{Si}(775)-\mathrm{Au}$ up to a 1 ML Au coverage, as predicted by our DFT calculations.

Computed RAS spectra are shown in Fig. 3(b) for the dbl$\mathrm{Au}(0.32 \mathrm{ML})$ and $\beta-\sqrt{3}(0.96 \mathrm{ML})$ models. As the RAS has been measured at room temperature, and spin-polarized states are only expected at low temperatures [34,35], we neglect spin effects in the calculations. For the dbl-Au case, both PBE and LDA functionals were tested, as RAS is known to be sensitive to the precise geometry of the double Au-chain on $\mathrm{Si}(111)-\mathrm{Au}$ [31]. In fact, the dimerization $d\left(d=\left(a_{1}-a_{0}\right) / a_{0}\right.$, where $a_{1}\left(a_{0}\right)$ is the maximum (undimerized) Au-Au distance along [110]) is found to be 0.11 within PBE compared with 0.19 in LDA. As shown in Fig. 3(b), the PBE spectrum is in good agreement with experiment across the whole energy range, regarding both the line shape and the intensity. The LDA spectrum differs mostly below $1.5 \mathrm{eV}$. However, the experimental reality probably corresponds to a situation intermediate to the LDA and PBE spectra. This is reasonable as the experimental lattice constant of $\mathrm{Si}$ (5.43 $\AA$ ), which defines the cell size and $\mathrm{Au}-\mathrm{Au}$ distances, lies almost midway between the LDA (5.40 $\AA$ ) and PBE (5.47 $\AA$ ) values. Thus, the calculated and measured RAS offer firm support for the dbl-Au structure at low Au coverages.

At higher coverages, the $\beta-\sqrt{3}$ signal follows the trends observed in the experiment. The whole spectrum is broadened and flattened with respect to the $\times 2$ phase, confirming the quenching of signals coming from the honeycombs. Lowenergy structures are also considerably weakened. In addition, the features around $E_{1}$ and $E_{2}$ are similarly shifted close to the baseline. By way of comparison, we also plot in Fig. 3(c) the computed spectrum for the highest coverage model (0.56 ML) within a $\times 2$ periodicity [see Fig. 1(d)]. The spectrum is clearly in strong disagreement with the high-coverage experimental data. Besides the intense signals below $1.5 \mathrm{eV}$, sharp negative features persist in the $1.5-3.0 \mathrm{eV}$ range from HC-related
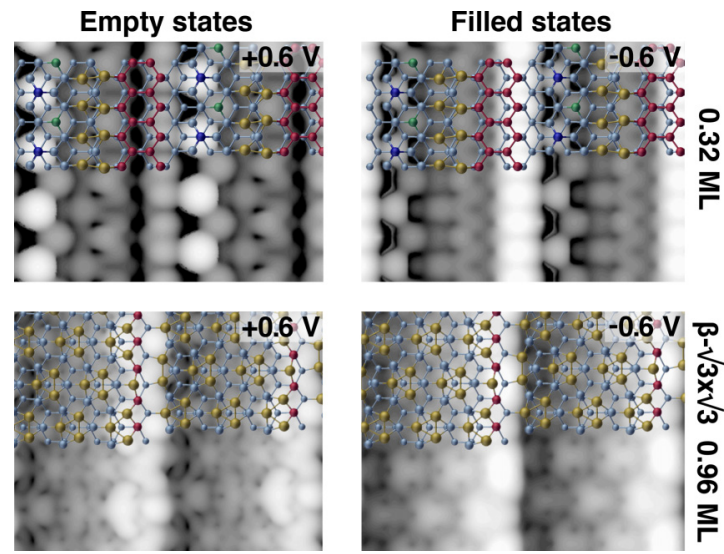

FIG. 4. Calculated STM images of Si(775)-Au with 0.32 ML and $0.96 \mathrm{ML}$ Au coverage for empty (left) and filled (right) states. The two uppermost layers of the respective structural motif are superimposed over the STM image for better clarification.

transitions, in stark contrast to the observed flat signal. These results therefore offer clear experimental support also for the $\beta-\sqrt{3}$ phase at higher coverages.

It should be mentioned, however, that our predicted models assume a fixed terrace width. Stronger morphological changes, in particular, the re-faceting of the surface for higher $\mathrm{Au}$ coverages [36], have not been considered. Indeed, a refaceting into $\sqrt{3}$-reconstructed $\mathrm{Si}(111)$-Au and compensating microfacets have been observed at a similar offcut [37]. Such a structure could also explain the LEED and RAS results, and is supported by the relative stability of our $\beta-\sqrt{3}$ model.

\section{Electronic properties}

In this section we consider the electronic properties of the $\mathrm{dbl}-\mathrm{Au}$ and $\beta-\sqrt{3}$ phases. Scanning tunneling microscopy (STM) images are simulated in order to allow for further experimental verification of the structural models predicted here. Figure 4 shows calculated empty- and filled-state STM images for a respective bias of $\pm 0.6 \mathrm{~V}$ for both the dbl-Au and $\beta-\sqrt{3}$ models. The present calculations for the dbl-Au model agree nicely with the findings in Ref. [9]. In particular the characteristic bright streaks and bright spots measured in the filled-state and empty-state STM, respectively, are well reproduced. They are traced to the $\mathrm{HC}$ step edge and the $\mathrm{Si}$ adatoms, respectively. The STM images of the $\beta-\sqrt{3}$ model are expected to deviate significantly from the dbl-Au model. The $(\sqrt{3} \times \sqrt{3})$ subpattern on top of terraces with $(1 \times 3)$ periodicity is quite visible in the present STM simulations. At the step edge a pattern with threefold periodicity is obtained, indicating a variation of the dangling bond filling along the step edge.

Figure 5 shows the calculated band structures for the dbl-Au and $\beta-\sqrt{3}$ models. To allow a better comparison with angle-resolved photoemission spectroscopy (ARPES) data, the unfolded band structures referring to the primitive translational symmetry [38] are also shown. For the dbl-Au model, the band derived from the rest atom's dangling bonds (green in Fig. 5) is about half filled and strongly dispersive along the chain direction, while the band formed by the adatom's dangling 

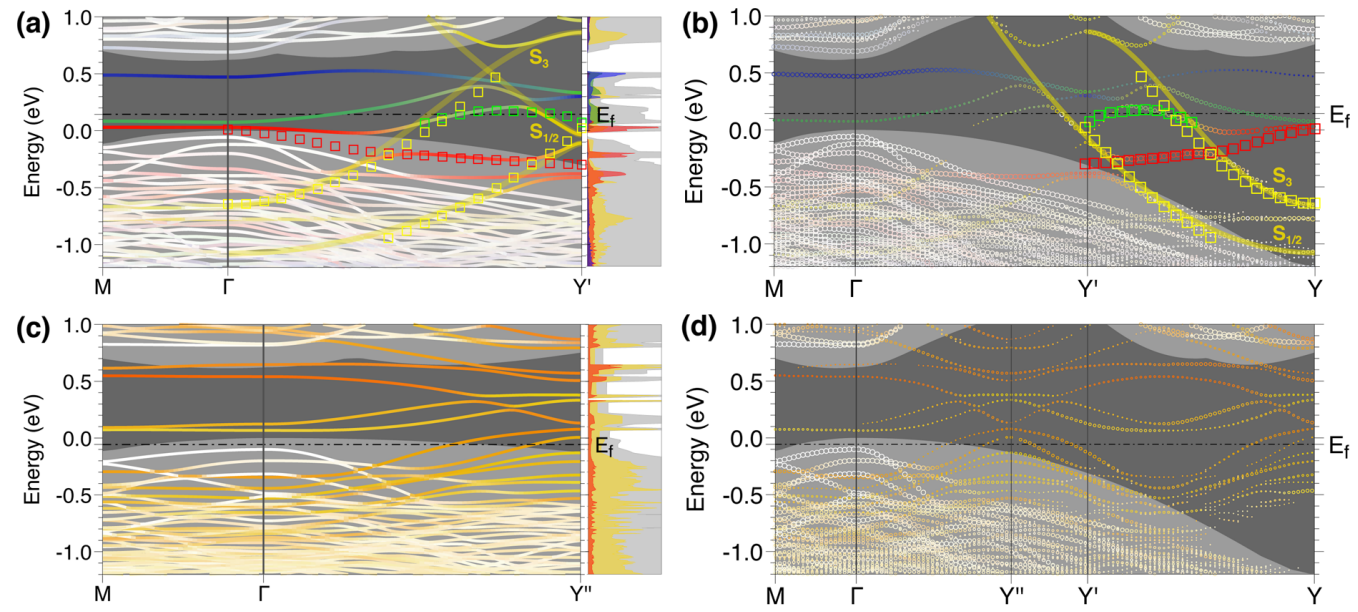

(e)

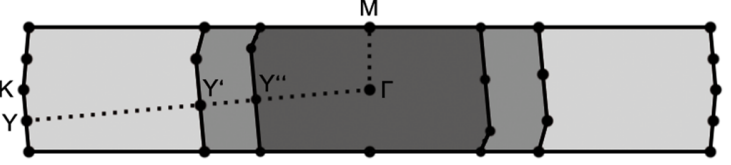

FIG. 5. Calculated band structures and density of states for the $\mathrm{Si}(775)-\mathrm{Au}$ dbl-Au (a) and $\beta-\sqrt{3}$ model (c). The corresponding unfolded bands for the primitive translational symmetry are shown in (b) and (d). The Brillouin zone notation is indicated in (e). The color coding of the bands/states corresponds to Fig. 1 and indicates localization at Au atoms (yellow), Si HC atoms (red), Si adatoms (blue), and Si rest atoms (green). The point size in the unfolded band structures represents the $\times 1$ character of the respective state; cf. Ref. [38]. Yellow squares indicate ARPES data from Ref. [10]. Yellow semitransparent lines are a fit to the Au bands with a model of free electrons and two energy levels (for more details see Refs. [24,39]).

bonds (blue in Fig. 5) is empty. There are several Au-related bands (yellow in Fig. 5), some of which (namely, $S_{1,2}$ and $S_{3}$ ) are well suited to explain the ARPES data [10]. They feature a parabolic dispersion along the Au-chain direction and cause-in conjunction with the rest atom band-a pronounced one-dimensional character of the dbl-Au electronic structure. The $S_{1}$ and $S_{2}$ bands are degenerate in the present calculation, but can be expected to split due to the Rashba effect [40].

The calculations for the dbl-Au model predict an occupation of roughly 2.5 electrons in the $S_{1,2}$ bands: 2 electrons in the lower branch and 0.5 electrons in the backfolded branch. The $S_{3}$ band hosts 1 electron. Altogether, 3.5 electrons can thus be assigned to the four $\mathrm{Au}$ atoms, corresponding to a net charge flow of 0.5 valence electrons from Au to the Si surface. Together with 1.5 electrons from the adatom and rest atom this provides 2 electrons that saturate the step edge atoms: The step-edge-related bands (red in Fig. 5) are completely filled and below the Fermi energy. This agrees with the findings by Aulbach et al. [9] and is in marked contrast to Si(557)-Au and $\mathrm{Si}(553)-\mathrm{Au}$, where every second or third Si step edge atom exhibits a half-filled dangling bond $[7,8]$.

The band structure of the $\beta-\sqrt{3}$ model is fundamentally different from the dbl-Au model. Most of the bands above and below the Fermi energy can be associated with Au atoms. The step edge states (colored red in Fig. 5) hybridize with Au states (orange colored bands). As these bands are also the only bands that cross the Fermi energy and are dispersive along the edge direction, we expect the charge transport to be mediated by step edge atoms and to be highly anisotropic, despite the high and 2D-like Au coverage.

\section{Spin order}

The $\mathrm{Si}(553)-\mathrm{Au}$ and $\mathrm{Si}(557)-\mathrm{Au}$ surfaces are currently the focus of intensive study due to their ability to host onedimensional spin chains [7,34,41-43]. Spins are reported to be located at the step edges and to order antiferromagnetically. Recently it was proposed that the magnetic exchange interactions at $\mathrm{Si}(553)-\mathrm{Au}$ are frustrated between neighboring spin chains, which in the framework of the anisotropic triangular Heisenberg model would stabilize an exotic 2D quantum spin liquid [8]. In the present work, the energetics of spin-ordered states at $\mathrm{Si}(775)-\mathrm{Au}$ is explored. All dangling bonds, including those on the terraces, were probed with respect to the stability of spin-polarized states. It is found that the dbl-Au model, in contrast to the $\beta-\sqrt{3}$ structure, indeed gives rise to stable spin-polarized configurations as shown in Fig. 6. In both cases the spins are located at the rest atom dangling bonds and order ferromagnetically or antiferromagnetically. In the
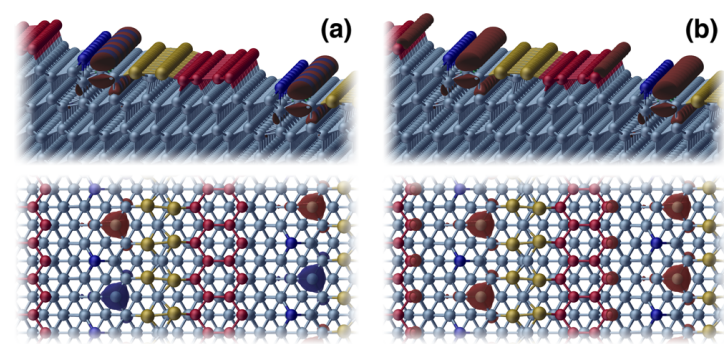

FIG. 6. Calculated spin density for (a) antiferromagnetic and (b) ferromagnetic spin ordered configurations of the dbl-Au model. Red and blue isosurfaces represent different spin orientations. 
TABLE I. Calculated energy of the antiferromagnetic and ferromagnetic spin ordered configurations of the dbl-Au model with respect to the non-spin-polarized surface.

\begin{tabular}{lc}
\hline \hline Spin configuration & Total energy $(\mathrm{meV}$ per $1 \times 2$ cell) \\
\hline Nonpolarized & 0.0 \\
Antiferromagnetic & -110.4 \\
Ferromagnetic & -108.8 \\
\hline \hline
\end{tabular}

case of the ferromagnetic (FM) ordering, minor magnetization density occurs also at the step edge. This is very different from $\mathrm{Si}(553)-\mathrm{Au}$ and $\mathrm{Si}(557)-\mathrm{Au}$, where the spin-polarized states are fully located at the step edge. The close proximity of the spin-split states to the atomic wires observed here for $\mathrm{Si}(775)$-Au possibly allows for spin manipulation and could be interesting for applications.

Compared to the non-spin-polarized dbl-Au model, the spin-polarized configurations are stabilized by about $0.1 \mathrm{eV}$ per $(1 \times 2)$ surface unit cell; cf. Table I. On the one hand, this is sufficiently large to stabilize these configurations at low temperatures. On the other hand, the energy gain due to spin order is small compared to the energy differences between various structural models and different stoichiometries and thus is not expected to affect the surface phase diagram shown in Fig. 2. Calculations with $(1 \times 6)$ surface unit cells show that the energy gain due to spin polarization is numerically robust with respect to the surface periodicity, at least for the FM case. The antiferromagnetic (AFM) spin-ordered configuration is only marginally more stable than the FM configuration, which indicates small coupling between neighboring spins. Perpendicular to the step edge, the distance between the spins, i.e., the $\mathrm{Si}$ rest atoms, is $21.5 \AA$ while in the parallel direction it is $7.7 \AA$. This is below the minimum spin-spin distance at $\mathrm{Si}(553)-\mathrm{Au}$, which amounts to $11.6 \AA$ A In the latter case a coupling parameter $J_{\|}=16 \mathrm{meV}$ was determined [8]. The AFM-FM energy difference of about $2 \mathrm{meV}$ calculated here is comparatively small and suggests the formation of adatom localized magnetic moments rather than ordered spin chains.

From the magnetization densities of the FM/AFM configurations it is found that the $\mathrm{Si}$ rest atom dangling bond is filled with a single unpaired electron. This contrasts with the non-spin-polarized configuration, where the rest atom dangling bond is filled with 0.5 electrons. This difference is also reflected in the FM/AFM band structures shown in Fig. 7 .

The Au-derived bands for the spin-polarized configurations are slightly raised and host only little more than 3 electrons, compared to the 3.5 electrons for the non-spin-polarized surface. The spin polarization thus allows a charge transfer from the Au to the Si rest atoms.

The non-spin-polarized band structure of the dbl-Au model (Fig. 5) indicates a strong contribution of Si rest atom states as well as Au states to the charge transport in the wire direction. This changes upon considering spin polarization. Here the rest atom states are shifted above the Fermi energy.

The spin-polarized calculations were performed for a surface periodicity of $(1 \times 4)$, leading to an additional band folding in the Au chain direction. Additional nondispersive gold and step edge bands are mapped onto the $M \Gamma$ path. Two
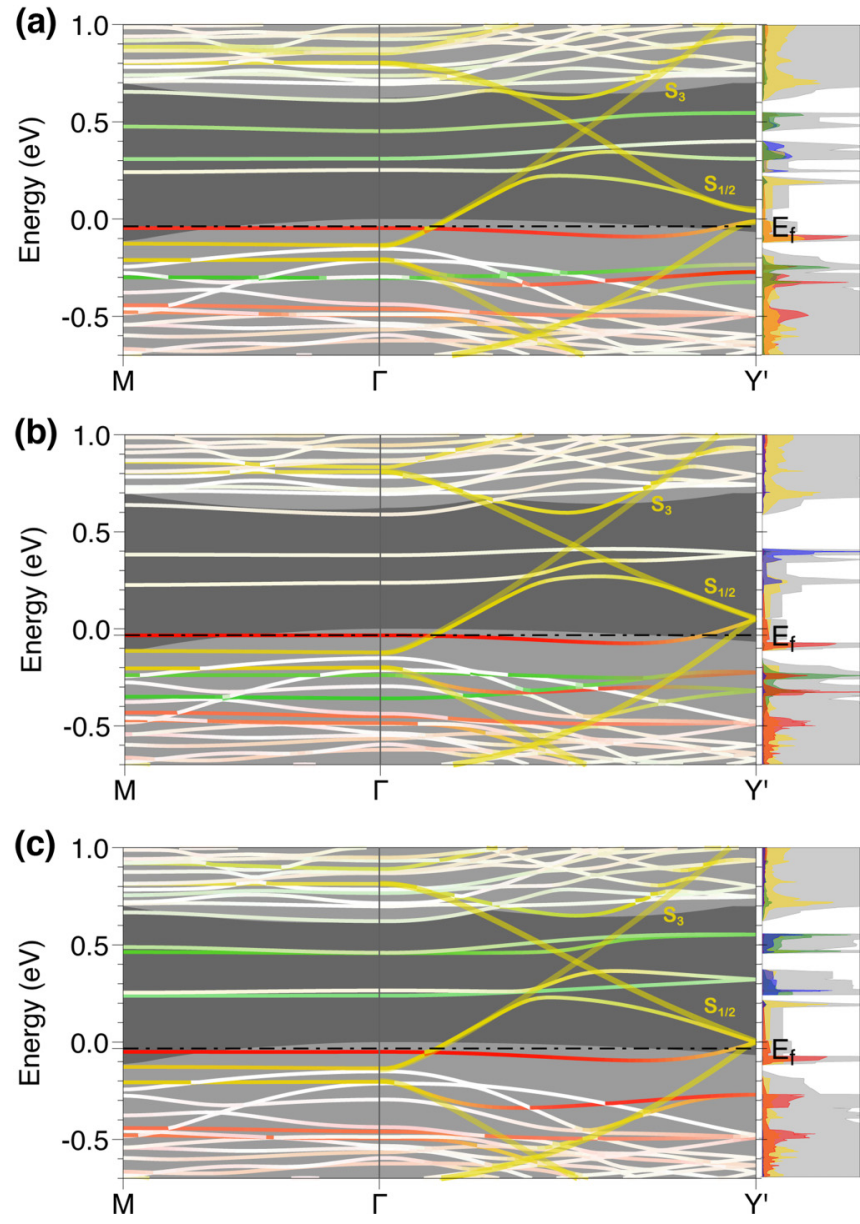

FIG. 7. Calculated band structures and density of states for the Si(775)-Au dbl-Au model: (a) AFM configuration; (b), (c) the two spin channels of the FM configuration. The color coding of the bands/states corresponds to that of Figs. 1 and 5, apart from the adatom-related bands, which are not colored for better visibility.

rest atom bands belonging to the two rest atoms per supercell can be identified for each configuration. In case of the AFM spin-up channel shown in Fig. 7(a), one of these bands is filled while the other is empty. The band structure of the spin-down channel looks exactly the same, except that the assignment of the rest atoms and bands is reversed. On the other hand, the spin-up channel of the FM configuration in Fig. 7(b) exhibits two rest atom bands which are completely filled. Here, the two bands cannot be identified with one of the rest atoms, but each atom equally contributes to each band. The band structure of the spin-down channel in Fig. 7(c) differs primarily in the two empty rest atom bands, leading to the ferromagnetic pattern. Another small difference lies in a slightly raised step edge band. The maximum of the band at the $\Gamma$ point now matches the Fermi energy, resulting in a not completely filled band for the spin-down channel. This explains the small amount of magnetization at the step edge illustrated in Fig. 6(b).

Notably, we find that the non-spin-polarized band structure shown in Fig. 5 describes the available ARPES data [10] better than the spin-polarized calculations shown in Fig. 7. Temperature effects that destroy the spin order could be a possible explanation $[34,35]$. 
No stable spin-polarized configuration of the $\beta-\sqrt{3}$ surface was found in the present calculations. This is partially related to the fact that no rest atom and adatoms are present on the surface. Only the Si step edge atoms and the Au atoms are left for possible spin polarization. These, however, show no indication of spin order, even though bands crossing the Fermi energy can be associated with these atoms and STM suggest different occupations for the rest atoms' dangling bonds.

\section{CONCLUSIONS}

Density functional theory calculations and reflectance anisotropy measurements indicate the existence of two energetically stable Au-stabilized reconstructions on the $\operatorname{Si}(775)$ surface. RAS, STM images, and electronic bands calculated for the low-coverage dbl-Au model agree well with the experimental data. It is found that Si rest atom dangling bonds of the dbl-Au model host an antiferromagnetic spin chain. Our calculations also predict for Au-rich preparation conditions the existence of a $\mathrm{Si}(775)-\mathrm{Au}$ structure with a $\beta-\sqrt{3}$ substructure, which is confirmed by RAS measurements. This $\beta-\sqrt{3}$ model does not show spin-ordered surface states.

\section{ACKNOWLEDGMENTS}

The authors gratefully acknowledge financial support by the Deutsche Forschungsgemeinschaft (FOR1700). VASP calculations were performed at the Paderborn Center for Parallel Computing $\left(\mathrm{PC}^{2}\right)$ and the High Performance Computing Center Stuttgart (HLRS). C.H. acknowledges the CINECA award under the ISCRA initiative for the availability of high performance computing resources and support, as well as HPC resources and support from the North-German Supercomputing Alliance (HLRN). S.C. and N.E. acknowledge the Ministerium für Innovation, Wissenschaft und Forschung des Landes Nordrhein-Westfalen, the Senatsverwaltung für Wirtschaft, Technologie und Forschung des Landes Berlin, and the Bundesministerium für Bildung und Forschung. We thank Eugen Speiser for help with the use of his RAS setup.
[1] T. Frigge, B. Hafke, T. Witte, B. Krenzer, C. Streubühr, A. S. Syed, V. M. Trontl, I. Avigo, P. Zhou, M. Ligges, D. von der Linde, U. Bovensiepen, M. Horn-von Hoegen, S. Wippermann, A. Lücke, S. Sanna, U. Gerstmann, and W. G. Schmidt, Nature (London) 544, 207 (2017).

[2] C. Zeng, P. R. C. Kent, T.-H. Kim, A.-P. Li, and H. H. Weitering, Nat. Mater. 7, 539 (2008).

[3] E. Jeckelmann, S. Sanna, W. G. Schmidt, E. Speiser, and N. Esser, Phys. Rev. B 93, 241407 (2016).

[4] H. W. Yeom, D. M. Oh, S. Wippermann, and W. G. Schmidt, ACS Nano 10, 810 (2016).

[5] C. Blumenstein, J. Schäfer, S. Mietke, S. Meyer, A. Dollinger, M. Lochner, X. Y. Cui, L. Patthey, R. Matzdorf, and R. Claessen, Nat. Phys. 7, 776 (2011).

[6] S. Cheon, T.-H. Kim, S.-H. Lee, and H. W. Yeom, Science 350, $182(2015)$

[7] S. C. Erwin and F. J. Himpsel, Nat. Commun. 1, 1 (2010).

[8] B. Hafke, T. Frigge, T. Witte, B. Krenzer, J. Aulbach, J. Schäfer, R. Claessen, S. C. Erwin, and M. H.-v. Hoegen, Phys. Rev. B 94, 161403(R) (2016).

[9] J. Aulbach, S. C. Erwin, R. Claessen, and J. Schäfer, Nano Lett. 16, 2698 (2016).

[10] J. N. Crain, J. L. McChesney, F. Zheng, M. C. Gallagher, P. C. Snijders, M. Bissen, C. Gundelach, S. C. Erwin, and F. J. Himpsel, Phys. Rev. B 69, 125401 (2004).

[11] S. C. Erwin, I. Barke, and F. J. Himpsel, Phys. Rev. B 80, 155409 (2009).

[12] S. G. Kwon and M. H. Kang, Phys. Rev. Lett. 113, 086101 (2014).

[13] G. Kresse and J. Furthmueller, Phys. Rev. B 54, 11169 (1996).

[14] J. P. Perdew, K. Burke, and M. Ernzerhof, Phys. Rev. Lett. 77, 3865 (1996)

[15] P. E. Blöchl, Phys. Rev. B 50, 17953 (1994).

[16] J. Tersoff and D. R. Hamann, Phys. Rev. Lett. 50, 1998 (1983).

[17] A. Marini, C. Hogan, M. Grüning, and D. Varsano, Comput. Phys. Commun. 180, 1392 (2009).
[18] P. Giannozzi, S. Baroni, N. Bonini, M. Calandra, R. Car, C. Cavazzoni, D. Ceresoli, G. L. Chiarotti, M. Cococcioni, I. Dabo, A. Dal Corso, S. de Gironcoli, S. Fabris, G. Fratesi, R. Gebauer, U. Gerstmann, C. Gougoussis, A. Kokalj, M. Lazzeri, L. Martin-Samos, N. Marzari, F. Mauri, R. Mazzarello, S. Paolini, A. Pasquarello, L. Paulatto, C. Sbraccia, S. Scandolo, G. Sclauzero, A. P. Seitsonen, A. Smogunov, P. Umari, and R. M. Wentzcovitch, J. Phys.: Condens. Matter 21, 395502 (2009).

[19] F. Manghi, R. D. Sole, A. Selloni, and E. Molinari, Phys. Rev. B 41, 9935 (1990).

[20] W. G. Schmidt, Phys. Status Solidi B 242, 2751 (2005).

[21] M. Krawiec, Phys. Rev. B 81, 115436 (2010).

[22] R. Seiwatz, Surf. Science 2, 473 (1964).

[23] F. Hötzel, K. Seino, S. Chandola, E. Speiser, N. Esser, F Bechstedt, and A. Pucci, J. Phys. Chem. Lett. 6, 3615 (2015).

[24] C. Braun, First principles investigation of spin ordering in nanowires, master's thesis, Universität Paderborn, 2016.

[25] T. Kadohira, J. Nakamura, and S. Watanabe, e-J. Surf. Sci. Nanotechnol. 2, 146 (2004).

[26] C. H. Patterson, S. Banerjee, and J. F. McGilp, Phys. Rev. B 94, 165417 (2016).

[27] G.-X. Qian, R. M. Martin, and D. J. Chadi, Phys. Rev. Lett. 60, 1962 (1988).

[28] G.-X. Qian, R. M. Martin, and D. J. Chadi, Phys. Rev. B 38, 7649 (1988).

[29] S. Chandola, K. Hinrichs, M. Gensch, N. Esser, S. Wippermann, W. G. Schmidt, F. Bechstedt, K. Fleischer, and J. F. McGilp, Phys. Rev. Lett. 102, 226805 (2009).

[30] C. Hogan, N. McAlinden, and J. F. McGilp, Phys. Status Solidi B 249, 1095 (2012).

[31] C. Hogan, E. Ferraro, N. McAlinden, and J. F. McGilp, Phys. Rev. Lett. 111, 087401 (2013).

[32] C. Hogan, E. Speiser, S. Chandola, S. Suchkova, J. Aulbach, J. Schäfer, S. Meyer, R. Claessen, and N. Esser (unpublished).

[33] N. McAlinden and J. F. McGilp, Europhys. Lett. 92, 67008 (2010). 
[34] S. C. Erwin and P. C. Snijders, Phys. Rev. B 87, 235316 (2013).

[35] D. Sánchez-Portal, S. Riikonen, and R. M. Martin, Phys. Rev. Lett. 93, 146803 (2004).

[36] L. Pedri, L. Toppozini, and M. C. Gallagher, Surf. Sci. 601, 924 (2007).

[37] L. Seehofer, S. Huhs, G. Falkenberg, and R. L. Johnson, Surf. Sci. 329, 157 (1995).

[38] P. V. C. Medeiros, S. Stafström, and J. Björk, Phys. Rev. B 89, 041407 (2014).
[39] N. Ashcroft and N. Mermin, Solid State Physics, HRW International Editions (Holt, Rinehart, and Winston, 1976).

[40] U. Gerstmann, N. J. Vollmers, A. Lücke, M. Babilon, and W. G. Schmidt, Phys. Rev. B 89, 165431 (2014).

[41] S. Polei, P. C. Snijders, S. C. Erwin, F. J. Himpsel, K. H. MeiwesBroer, and I. Barke, Phys. Rev. Lett. 111, 156801 (2013).

[42] S. Polei, P. C. Snijders, K. H. Meiwes-Broer, and I. Barke, Phys. Rev. B 89, 205420 (2014).

[43] J. Aulbach, J. Schäfer, S. C. Erwin, S. Meyer, C. Loho, J. Settelein, and R. Claessen, Phys. Rev. Lett. 111, 137203 (2013). 